\title{
Using of a cost-based Unit Commitment algorithm to assist bidding strategy decisions
}

\author{
A. Borghetti, A. Frangioni, F. Lacalandra, C.A. Nucci, P. Pelacchi
}

\begin{abstract}
The paper describes a procedure developed to assist a generating company in choosing the most convenient bidding strategies for a day-ahead electricity energy market. According to the proposed method, the profit maximization problem is transformed into a minimization problem that can be solved by a traditional hydro-thermal unit commitment program after implementing a few modifications. The paper describes the modifications introduced in a unit commitment program based on the Lagrangian relaxation approach and on a disaggregated Bundle method for the solution of the dual problem. It also presents some results obtained for a realistic data set of hydrothermal power plants. The results are discussed in order to emphasize how the method can be applied to assess the bidding strategy choice of a given company.
\end{abstract}

Index Terms-Unit commitment, electricity market, bidding strategies.

\section{NOMENClATURE}

$\mathbf{p}_{H}$

$\frac{p_{i}}{\bar{p}_{i}} \bar{p}_{h}$
$\bar{\tau}_{i}^{u}, \tau_{i}^{d}$
$c_{i, t}$
$s_{i, t}^{u}$

$|H|$-rows $|T|$-columns matrix, whose rows are the $|T|$-dimensional arrays $\mathbf{p}_{h}$ of production levels $p_{h, t}$ of hydro unit $h$ during each period $t$. minimum/maximum outputs of the units. minimum up- and down-times of thermal units. per hour operating cost of committed thermal unit $i$ at period $t$, as a quadratic function of production level $p_{i, t}$. unit $i$ is committed at the beginning of period $t$. It can depend on the number of periods that the unit has been down.

$w_{h, t}, s_{h, t}, a_{h, t}$ water discharge rate and spillage of hydro unit $h$ and net inflow rate of its reservoir during period $t$, respectively.

$n_{t} \quad$ length of period $t$. Summation $\sum_{t \in T} n_{t}$ is equal to the optimization horizon.

$V_{h, t} \quad$ storage volume of the reservoir of hydro unit $h$ at the end of period $t$, limited between a maximum $\bar{V}_{h}$ and a minimum $\underline{V}_{h}$ value.

$V_{h, \text { in }}, V_{h, \text { end }} \quad$ storage volumes of the reservoir of hydro unit $h$ at the beginning and at the end of the optimization horizon, as given by a long-term hydro-scheduling.

\section{INTRODUCTION} ( $|T|$ : number of time periods ; $t$ : time period index).

D $\quad|T|$-dimensional vector of load demands $D_{t}$ in each period $t$.

$\mathbf{u} \quad|I|$-rows $|T|$-columns matrix, whose rows are the $|T|$-dimensional arrays $\mathbf{u}_{i}$ of the $0-1$ variables $u_{i, t}$ indicating the commitment state of thermal unit $i$ during period $t$.

$\mathbf{p}_{I} \quad|I|$-rows $|T|$-columns matrix, whose rows are the $|T|$-dimensional arrays $\mathbf{p}_{i}$ of production levels $p_{i, t}$ of thermal unit $i$ during each period $t$.

This work was supported in part by the Italian National Research Council under Grant CNRC00C821.

A. Borghetti, F. Lacalandra and C. A. Nucci are with the Department of Electrical Engineering, University of Bologna, 40136 Bologna, Italy.

A. Frangioni is with the Department of Computer Science, University of Pisa, 56125 Pisa, Italy.

P. Pelacchi is with the Department of Electrical Systems and Automation, University of Pisa, 56122 Pisa, Italy.

\section{$\mathrm{F}$}

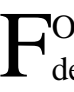
OR many years, most electric power utilities have developed and used optimization packages to solve the cost-based unit commitment problem relevant to their specific park of hydro-thermal generating units over a daily or weekly horizon. Nowadays, however, in the framework of some competitive electricity markets, commitment decisions are made by a schedule coordinator (power exchange) on the basis of bids presented by the market participants (generating companies and consumption companies) [1].

One of the most important problems that the generating companies have to face in this scenario is how to identify the most convenient bids, i.e. the bidding strategy that allows maximizing the profit of the company.

A considerable number of studies have been recently carried out on this subject (e.g. [2-18]).

In general, the above studies propose new optimization models to accomplish the problem, namely to determine the most convenient behavior of the market participants, and 
present new algorithms for the solutions of these models (e.g. [5,6,13-15]).

The aim of this paper is, instead, to investigate how traditional cost-based unit commitment tools, which are already available to generating companies, can be used in the competitive electricity market for the problem of interest. In particular, the paper will show the framework in which a traditional cost-based unit commitment tool can be used to assist bidding strategy decisions to a day-ahead electricity pool market. The optimization model used in this paper is aimed to be independent, as far as possible, of the specific power exchange rules, which appear to be in perpetual evolution.

The structure of the paper is as follows: Section III briefly reviews the basic problem on how to choose the most convenient bidding strategy by a generating company in an energy market, taking into account its potential capability of altering market-clearing prices. Section IV presents the proposed formulation of the optimal bidding strategy problem in which the optimization problem is adjusted in order to be solved by a cost-based unit commitment package after a few suitable modifications. Section V presents the proposed modifications, that we have here introduced in a cost-based unit commitment program - described in [19] - based on the Lagrangian relaxation approach and on a disaggregated Bundle method for the solution of the dual problem. In Section VI some results, obtained for a realistic data set of hydrothermal power plants, are shown. These results are discussed in order to show how the method can be applied to assess the bidding strategy choice of a given company.

\section{GENERAL THEORY}

We suppose that in each period $t$ of the optimization horizon $T$, which covers one or more energy market sessions, the generating company will offer bidding curves with the aim of maximizing its profit. The profit is given by the difference between the total revenue and the total costs: economic theory indicates that the equilibrium, i.e. the condition of maximum profit, is reached when the value of the marginal revenue $(M R)$ is equal to the value of the marginal costs $(M C)$, assuming the market price $(M P)$ to be above the level of the average variable cost $(A V C)$. Note that, without the presence of other constraints, it is not convenient for a company to produce when $M P$ is lower than $A V C$. The variable costs are those usually considered in unit commitment problems, i.e., the thermoelectric production costs associated with the fuel consumption, the start-up (and shut-down) costs of the thermoelectric units and the opportunity costs associated with the use of water for hydroelectric production. The variable cost $(V C)$ can be formulated as:

$$
V C=\sum_{i \in I} \sum_{t \in T}\left[u_{i, t} \cdot c_{i, t}\left(p_{i, t}\right)+s_{i, t}^{u}\left(\mathbf{u}_{i}\right)\right]
$$

taking into account the physical and operating constraints of the generation units. For example the usual operating constraints for each thermal unit $i$ and time period $t$ are $u_{i, t} \cdot \underline{p}_{i} \leq p_{i, t} \leq u_{i, t} \cdot \bar{p}_{i}$

$\tau_{i}^{d}$ and $\tau_{i}^{u}$ constraints.

If the time step is chosen particularly small with comparison of the slow dynamical response of some thermal power plants, the outputs of slow unit $i$ is limited by the socalled ramp-rate constraints:

$$
\Delta_{i}^{d} \leq p_{i, t}-p_{i, t-1} \leq \Delta_{i}^{u} \quad \forall t \in T
$$

where $\Delta_{i}^{d}$ and $\Delta_{i}^{u}$ are the maximum decrease and increase, respectively, in the output of unit $i$ in one time period.

The generation of hydroelectric power plants reduces the power demand that must be fulfilled by the costly thermal production. The electrical output of a hydro power plant depends on the water discharge, the head (which can be expressed as a function of the reservoir volume) and the efficiency of the hydraulic turbine (which is also a function of the water discharge and the head). The operating points are restricted by minimal and maximal water discharges. If $p_{h, t}$ is assumed to be a linear function of water discharge $w_{h, t}$ and independent of the water head, an assumption frequently considered reasonable in the literature on the subject, hydro production can be represented by a simplified model which, for each hydro unit $h$ of reservoir network $b$ and for each time period $t$, takes into account the hydraulic continuity equations to compute the reservoir storages, the storage, discharge rate and spillage limits, and the initial and ending storage constraints:

$$
\begin{aligned}
& V_{h, t}-V_{h, t-1}=n_{t} \cdot\left[\left(a_{h, t}-w_{h, t}-s_{h, t}\right)+\sum_{h^{\prime} \in H_{h}^{\prime}}\left(s_{h^{\prime}, t-\tau_{h^{\prime}, h}}+w_{h^{\prime}, t-\tau_{h^{\prime}, h}}\right)\right] \\
& \underline{V}_{h} \leq V_{h, t} \leq \bar{V}_{h} \\
& \underline{S}_{h} \leq s_{h, t} \leq \bar{S}_{h} \\
& 0 \leq w_{h, t} \leq \bar{W}_{h} \\
& V_{h, \text { in }} \text { and } V_{h, \text { end }} \text { constraints }
\end{aligned}
$$

The model described by (4) includes an energy constraint since the energy produced by the hydro plants in a basin at the end of the market session $T$ is limited by the total amount of available water to be discharged in the optimization horizon.

As already mentioned, the above elements of the model are identical to those used for cost-based UC algorithms (eg. [19]). The main difficulty that arises in adapting the model to the competitive market case, in order to choose the most convenient bidding strategy, consists in evaluating the energy price at every time period: this is fundamental for computing the revenue of the producer, but it is subject to the market characteristics.

To address the problem, it is usual to distinguish the case of a company that does not have the capability to altering marketclearing prices (competitive company), therefore behaving as price-taker, from the case of a company having so-called market power (oligopolist) (e.g. [5,6,14,18]).

For the case of a competitive company, $M R$ is equal to $M P$ and is independent on the behavior of the company. The competitive company will choose the bidding strategy which 
allows reaching the most convenient production levels, i.e. the production level where $M C$ becomes equal to $M P$, being $M P$ greater than $A V C$. Note that the supply curve of a competitive company, i.e. the curve that relates the values of $M P$ and the values of the optimal production level, is defined as the part of the $M C$ curve that is above the $A V C$ curve.

For the case of an oligopolistic company, such a supply curve cannot, in general, be defined, as the production level depends on the elasticity of the demand and on the behavior of the competitors. However, the supply curve can be univocally defined if we know the elasticity of the demand and the supply curves of the competitors.

This is the case when the demand can be considered inelastic and the oligopolistic company is the only company with market power, the other ones behaving as price-takers (e.g. $[9,14])$. In fact, in such a case, a supply curve can be defined for the competitors by aggregating the $M C$ curves (above $A V C$ ) of the price-taker competitors. For each $M P$ value, the production level of the oligopolistic company is given by the difference between the forecasted demand level and the production level of the competitors. The supply curve of this unique oligopolistic company is subsequently defined.

\section{MODIFICATION OF A PROFIT MAXIMIZATION PROBLEM IN A COST MINIMIZATION PROBLEM}

The optimization problem that has to be solved to find the equilibrium that maximizes the profit of the company is

$$
\begin{aligned}
& \max _{\mathbf{u}, \mathbf{p}_{I}, \mathbf{p}_{H}}(R-V C)= \\
& \max _{\mathbf{u}, \mathbf{p}_{I}, \mathbf{p}_{H}}\left\{\sum_{t \in T}\left[M P_{t} \cdot\left(\sum_{i \in I} p_{i, t}+\sum_{h \in H} p_{h, t}\right)\right]-V C\right\}
\end{aligned}
$$

subject to the physical and operating constraints (2)-(4) of the generating plants.

We distinguish now between the cases of competitive and oligopolistic generating companies.

\section{A. Competitive generating company (price-taker)}

As already mentioned in Section III, the $M P_{t}$ values for each period $t$ are independent on the production level of the price-taker company.

Given that

$$
\sum_{i \in I^{\prime}} p_{i, t}+\sum_{h \in H^{\prime}} p_{h, t}=D_{t}-p_{\text {others }, t} \quad \forall t \in T
$$

where $D_{t}$ is the forecasted system load demand and $p_{\text {others }, t}$ is the production level of the competitors at period $t$, optimization problem (5), that has to be solved to find the most convenient production level, can be modified as

$$
\min _{\mathbf{u}, \mathbf{p}_{t}, \mathbf{p}_{H}, \mathbf{p}_{\text {others }}}\left[V C+\sum_{t \in T}\left(M P_{t} \cdot p_{\text {others }, t}\right)\right]
$$

subject to (6) and constraints (2)-(4).

Problem (7) can be interpreted as a cost-based unit commitment model of the generating company, modified with an additional unit representing the costs associated with the revenues of the competitors. Note that the cost function of this additional unit is linear. Its coefficient $M P_{t}$, however, changes at every time period $t$. These $M P_{t}$ values are input of the problem and must be forecasted by the company, on the basis of the specific electricity market.

\section{B. Oligopolistic company}

For the case of an oligopolistic company the $M P_{t}$ values depends on the bidding strategy and the production level of the company.

As already mentioned in Section III, if the company is the only price-maker of the market and the demand is inelastic, an aggregate supply curve of the competitors $p_{\text {others }, t}\left(M P_{t}\right)$ can be defined, for each time period $t$, by aggregating the $M C$ curves of the competitors. Therefore, we can define its inverse function $M P_{t}\left(p_{\text {others }, t}\right)$ that returns the expected market price value at time period $t$, for each aggregated level of production by all the price-taker companies. Using again (6) and changing sign, the optimization problem becomes

$\min _{\mathbf{u}, \mathbf{p}_{I}, \mathbf{p}_{H}, \mathbf{p}_{\text {others }}}\left\{V C+\sum_{t \in T}\left[M P_{t}\left(p_{\text {others }, t}\right) \cdot p_{\text {others }, t}-D_{t} \cdot M P_{t}\left(p_{\text {others }, t}\right)\right]\right\}$

subject to (6) and (2)-(4).

Optimization problem (8) can be seen as a cost-based unit commitment model with an additional unit whose production level $p_{\text {others }}$ refers to the production of the competitors and the "cost function"

$C_{t}\left(p_{\text {others }, t}\right)=M P_{t}\left(p_{\text {others }, t}\right) \cdot p_{\text {others }, t}-D_{t} \cdot M P_{t}\left(p_{\text {others }, t}\right)$

depends on their $M C$ curves and demand level $D$.

The parameters of such a cost function of the additional unit are therefore different in each time period $t$. Note that, given the functions $M P_{t}\left(p_{\text {others }, t}\right)$, optimization problem (9) is independent of the specific electricity market rules.

For the case of an oligopolistic company which is not the only price-maker in market, the problem of estimating the supply curve of the competitors arises. One possibility is to use historical market data to construct probabilistic curves $[6,20]$, turning (8) into a stochastic optimization problem. According to the hypotheses on the functions forms, such a problem could be solved by several different approaches, such as Monte Carlo methods [6], stochastic gradient methods [21] or decomposition algorithms, e.g. using Bundle methods [22]. Approaches based on game theory could also be used, but the characteristics of the problem (large scale, combinatorial) are not favorable for this kind of techniques. ${ }^{1}$

An efficient solution of the above-mentioned (large-scale mixed-integer non-linear) optimization problem can be obtained by using a cost-based unit commitment algorithm, with limited modifications related to the additional production unit that takes into account the competitors' presence. Then, the most convenient values of matrices $\mathbf{u}, \mathbf{p}$, and $\mathbf{h}$ are determined as well as - for the case of the oligopolistic company - the most convenient values of $M P$.

From these results a single bidding curve for all the

\footnotetext{
${ }^{1}$ In what follows, this paper will not consider the case of the presence of more than one oligopolistic generating company in the market.
} 
companies or a bidding curve for each power plant or section may be derived on the basis of the specific market rules. The goal of the bidding curve construction is to force the Power Exchanger outcomes to be closer to the calculated most convenient values of $M P$ and production levels. Some procedures have been proposed in the literature on the subject, e.g. $[7,15]$.

\section{SOLUTION METHODOLOGY BASED ON A LAGRANGIAN RELAXATION ALGORITHM}

A hydrothermal unit commitment solution methodology using Lagrangian heuristics based on disaggregated bundle methods has been presented in [19].

A significant advantage of the adoption of the Lagrangian relaxation algorithm is its ability to compute at the same time both lower and upper bound values for the problem, giving a clear indication of the quality of the obtained solution. Moreover, the Lagrangian relaxation algorithm allows, in general, including detailed system representation and modifications to the problem in a simpler way than it would be possible with other techniques.

In the following, we shall describe the specific modification introduced in the solution algorithm in order to solve problems (7) and (8) relevant to the competitive and oligopolistic one, respectively.

\section{A. Competitive generating company (price-taker)}

By rewriting problem (7) of a single competitive company, including (6) in order to eliminate variable $p_{\text {others }, \text {, we obtain a }}$ problem in which the hydro and thermal generating costs depends on the $M P_{t}$ values. The problem appears to be non separable with respect of the competitive company generating units, because of constraints $p_{\text {others }, t} \geq 0(\forall t)$. However, a single price taker company, by definition, could satisfy only part of the demand and therefore these constraints are always inactive. Thus, the price taker case can be solved at the cost of one Lagrangian iteration of the traditional Langragian relaxation based unit commitment algorithm, using the $M P_{t}$ values as the Lagrangian multipliers.

\section{B. Oligopolistic generating company (price-maker)}

The dual function of problem (8), obtained by the Lagrangian relaxation of the system demand requirement (6), through Lagrangian multipliers $\lambda_{t}$, becomes

$$
L(\boldsymbol{?})=\min _{\mathbf{u}, \mathbf{p}_{t}, \mathbf{p}_{H}}\left\{\begin{array}{l}
V C+\sum_{t \in T} C_{t}\left(p_{\text {others }, t}\right)+ \\
+\sum_{t \in T} \lambda_{t} \cdot\left(D_{t}-p_{\text {others }, t}-\sum_{i \in I} p_{i, t}-\sum_{b \in B} \sum_{h \in H_{b}} p_{h, t}\right)
\end{array}\right\}
$$

subject to constraints (2)-(4).

Dual function (10) exhibits a disaggregated structure, as the traditional cost-based UC model. In this paper, function $M P\left(p_{\text {others }}\right)$ is modeled by a piecewise linear function of the type

$$
\begin{aligned}
& M P_{t}\left(p_{\text {others }, t}\right)=a_{t}^{k}+b_{t}^{k} p_{\text {others }, t} \\
& \text { if } \quad \underline{p}_{\text {others }, t}^{k} \leq p_{\text {others }, t} \leq \bar{p}_{\text {others }, t}^{k}
\end{aligned}
$$

where $k=1 \ldots K$ and $\underline{p}_{\text {others }, t}^{k}, \bar{p}_{\text {others }, t}^{k}$ are, for each value of $k$, the minimum and maximum value of $p_{\text {others, } t}$, respectively.

The cost function of the additional unit becomes of the type

$C_{t}\left(p_{\text {others }, t}\right)=b_{t}^{k} p_{\text {others }, t}^{2}+\left(a_{t}^{k}-b_{t}^{k} D_{t}\right) p_{\text {others }, t}-a_{t}^{k} D_{t}$

if $\underline{p}_{\text {others }, t}^{k} \leq p_{\text {others }, t} \leq \bar{p}_{\text {others }, t}^{k}$

and the subproblem for the additional unit becomes:

$$
\begin{array}{lr}
L_{\text {other }}\left(\lambda_{t}\right)=\min _{k=1, \ldots, K} \min _{p_{\text {others }, t}}\left\{b_{t}^{k} p_{\text {others }, t}^{2}+\left(a_{t}^{k}-b_{t}^{k} D_{t}-\lambda_{t}\right) p_{\text {others }, t}+\right. \\
\left.-a_{t}^{k} D_{t}: \underline{p}_{\text {others }, t}^{k} \leq p_{\text {others }, t} \leq \bar{p}_{\text {others }, t}^{k}\right\} & \forall t \in T
\end{array}
$$

Such a problem is solved by the solution of $K \cdot t$ independent quadratic problems in one variable, one for each interval and time period, and therefore does not affect in a relevant way, even for large $\mathrm{K}$, the computational cost of solving the Lagrangian relaxation. Therefore, all algorithms for finding a good set of Lagrangian multipliers, such as those used in [19] and the many others proposed in the literature, can be used in this context. This allows to easily adapt existing codes for the solution of minimum cost unit commitment problems to the case of the maximum profit objective.

As in [19], the optimal values ?* of the Lagrange multipliers are obtained by the solution of the Lagrangian dual

$L^{*}=\max _{\mathbf{?} \geq \mathbf{0}} L(?)$.

by applying a bundle method. $L^{*}$ provides a lower bound on the optimal value of the objective function of (8), and the difference between these two values is called duality gap. As a by-product of the process of maximizing $L$, a schedule $\left\{\mathbf{u}, \mathbf{p}_{I}, \mathbf{p}_{H}, \mathbf{p}_{\text {others }}\right\}$ is obtained from the solution of problem (10) with $?=?^{*}$. In general, this schedule does not satisfy constraint (6) and, therefore, Lagrangian heuristic techniques for computing a near-optimal schedule are implemented, which suitably exploit the information made available by the use of the bundle method [19]. Note that, however, starting from the solution of the dual, a feasible solution of problem (8)

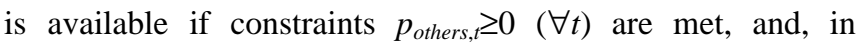
general, this is easier to obtain than in the case of the traditional cost-based unit commitment.

The above described approach holds when the units belonging to the competitive companies are sufficient to satisfy the load. If in a period some of the units of the oligopolistic company are needed to satisfy the system load (here considered fixed) the more convenient of the oligopolistic company offer would be infinite.

As shown in [16], the behavior of the oligopolistic company depends on the quota of the power plants belonging to it, on the shape of the $M P\left(p_{\text {others }}\right)$ function (on its steepness, in particular), and on the cost function of its units. The next section shows some of the results obtained by using the described approach for a realistic data set of hydro-thermal 
power plants.

\section{COMPUTATIONAL EXPERIENCE AND RESULTS}

The software tools described in the previous chapter have been used to perform a number of numerical studies to assess the characteristics of the proposed approach. Some of the obtained results are presented in this section. In particular, a reference scenario is described and the results obtained with the proposed approach, are compared with those obtained by applying the traditional cost-based unit commitment program.

\section{A. Description of system}

The reference scenario assumes a production power system formed by a set of thermal units, with total nominal power equal to $11634 \mathrm{MW}$ and hydro power plants with maximum generating power equal to the $40 \%$ of the thermal one (i.e., $4654 \mathrm{MW})$.

The thermoelectric unit parameters other than those of the operating cost functions are generated from the 10-unit data published in [23] by repeating 7 times the set of units. Parameters $a, b$ and $c$ of the cost functions $c_{i}\left(p_{i}\right)=a+b \cdot p_{i}+c \cdot p_{i}^{2}$ relevant of each of the 70 units are randomly generated according to normal distributions, whose mean values are equal to the relevant values of parameters $a, b$ and $c$ of [23] and standard deviations are equal to 100, 5 and 0.0001 for parameter $a$, parameter $b$ and parameter $c$, respectively.

Also the load profile is taken from [23], but the values are modified in order to obtain a margin at the load peak equal to $40 \%$ with respect of the total system production capacity (i.e., a peak load value equal to $9772 \mathrm{MW}$ ).

The energy available for the hydro units, in terms of water that has to be used in the optimization horizon, is set equal to $8260 \mathrm{MWh}$

The hydro and thermal units are allocated among different companies. One of the companies is assumed to have market power, the oligopolistic company, while the others behave as price takers and are indicated as the set of competitive companies.

\section{B. Description of the set of generating units of the oligopolistic company and of the competitive ones}

Results will be shown, relevant to cases that are characterized by the hypothesis that in each period the set of the units of the competitive companies is always sufficient to satisfy the system load demand.

In the reference case, the maximum generating power of the oligopolistic company is set equal to the $40 \%$ of the maximum power of all the set of generating units. Such a capacity is given by 25 of the 70 thermal units of the system and by hydro units with total maximum power equal to $40 \%$ of the thermal capacity of the oligopolistic company and with $40 \%$ of the energy stored in all basins of the system.

The results relevant to the case of an oligopolistic company with lower production capacity (namely 35\%, 30\%, 20\% and $14.3 \%$ of the total production capacity of the system) will be shown.

All the units not belonging to the oligopolistic company are assumed to belong to the set of competitive companies.

\section{Results for the case of competitive companies}

A traditional cost-based unit commitment of the entire set of generating groups of the reference system has been calculated. In what follows, such a cost-based unit commitment will be used as a point of reference. Moreover, the calculated vector of lagrangian multipliers $\lambda$ is used as input for the profit-based unit commitment of the generating groups belonging to the competitive companies, i.e. $\lambda$ is assumed to represent the vector of market prices MP.

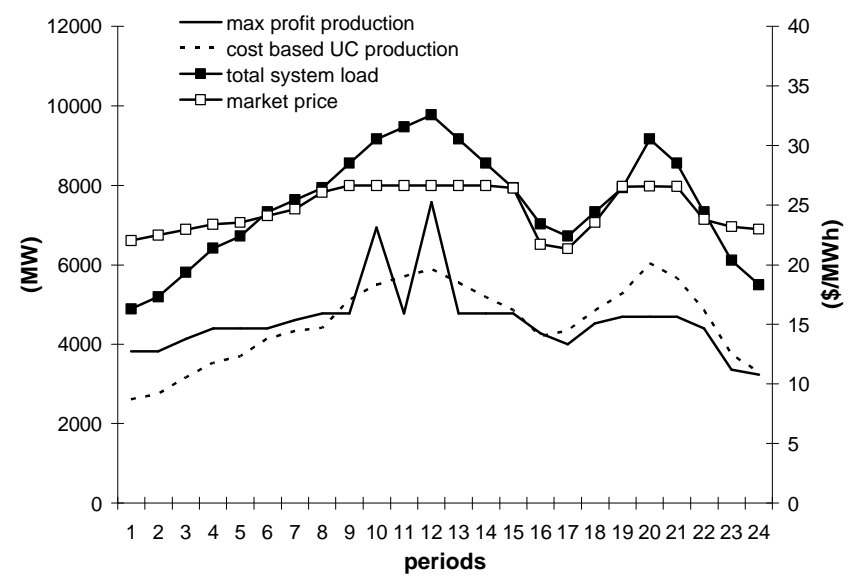

Fig. 1. Competitive company: comparison between the total production calculated with the maximum profit algorithm, whose input is the marginal price vector, and the traditional cost-based unit commitment approach, whose input is the system load profile, for the reference case.

Fig. 1 shows the comparison between the overall power production of the units of the competitive companies relevant to the profit maximization solution, and to the one calculated using the traditional cost-based unit commitment program. For most of the periods, the profile of the thermoelectric production, as well as the values of unit commitment matrix $\mathbf{u}$ of thermal units obtained for the two solutions, is similar in the two cases. Similar behaviors of the competitive companies production profile have been obtained in all the simulations carried out, apart from the case of competitive companies with thermoelectric units only, due to the impact of the adopted Lagrangian heuristics for the unit commitment of thermal units. The hydro power plants production is however different in the two cases, as in the profit maximization algorithm the production is driven by the price diagram, while in the traditional unit commitment the hydroelectric production is dispatched on the basis of the load profile.

In order to identify function $M P\left(p_{\text {others }}\right)$, necessary for the simulation of the oligopolistic company behavior, the maximum profit unit commitment of the units of the competitive companies has been repeated for various MP vectors, namely 7 different vectors obtained by using, as in Fig. 1, the values of $\lambda$ and the vectors whose values are $10 \%$ and $20 \%$ lower and $10 \%, 20 \%, 50 \%$ and $70 \%$ higher than those of $\lambda$. 
As an example, Fig. 2 shows the overall power production of the units of the competitive companies calculated for the 7 different MPs, relevant to two periods. On the basis of the obtained overall production levels, $a$ and $b$ coefficients of (11) are identified by means a least-square curve fitting. For the examined cases, a two segment function is considered to be adequate. The two periods are related to a period in which the hydro power plants are used (period 12) and a period in which only thermoelectric power plants are dispatched (period 5).
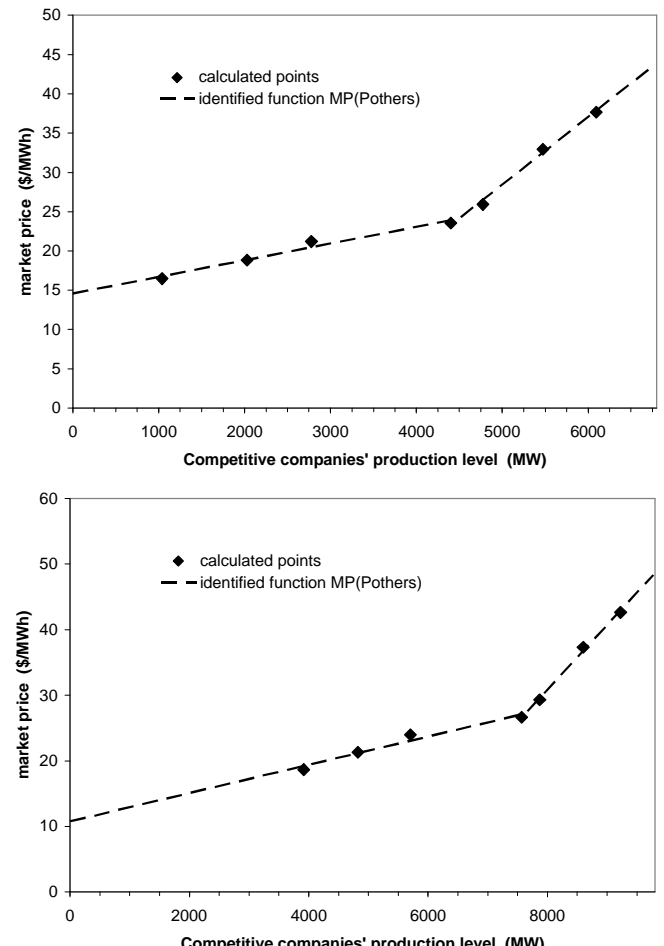

b)

Fig. 2. Competitive company: linear least-square identification of the values of the parameters of function $M P\left(p_{\text {others }}\right)$, at period 5 (a) and at period 12 (b). The points with white background are those calculated assuming MP equal the vector of lagrangian multipliers $\lambda$ evaluated by the traditional cost-based unit commitment.

\section{Results for the case of the oligopolistic company}

Identified functions $M P\left(p_{\text {others }}\right)$ have been used for the solution of the maximum profit unit commitment problem of the oligopolistic company, again for the reference system. Fig. 3 shows the comparison between the overall power production of the units of the oligopolistic company relevant to the profit maximization solution and that calculated by the traditional cost-based unit commitment program. Fig. 3 shows also the total system load, already shown in Fig. 1, and the market price.

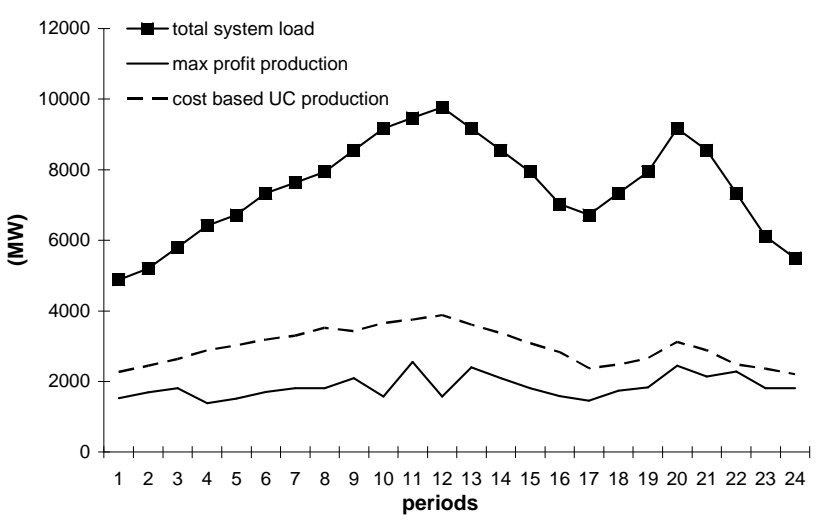

Fig. 3. Oligopolistic company: comparison between the total production calculated with the maximum profit algorithm and the traditional cost-based unit commitment approach. Also the system load profile is shown

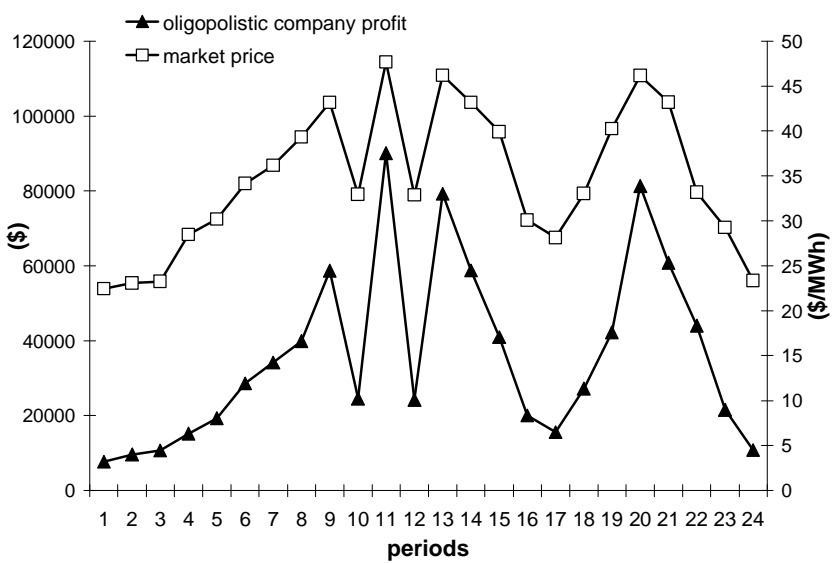

Fig. 4. Oligopolistic company: profit profile and market price profile calculated by using the identified function $M P\left(p_{\text {others }}\right)$.

Fig. 4 shows the oligopolistic company profit profile and the market price. The market price profile greatly differ from that of Fig. 1, due to the fact that in Fig. 1 the market price is set equal to the values of the Lagrange multipliers $\lambda$ given by the solution of the traditional cost-based unit commitment, while the market price shown in Fig. 4 is given by the identified functions $M P\left(p_{\text {others }}\right)$. The market price of Fig. 4 are greater than those of Fig. 1, as, as expected, the maximum profit behavior of the oligopolistic company results in lower power production levels at each period, as also shown in Fig. 4. The price behavior is greatly influenced by the $M P\left(p_{\text {others }}\right)$ functions. For example in periods 10 and 12 we see that the price falls, as the competitors presents a flat MP curve, due to the use of the hydro units. 


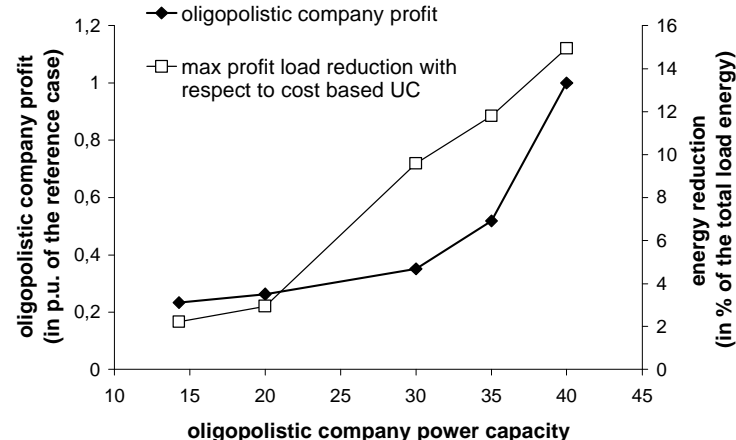

a)

(in \% of the total capacity)

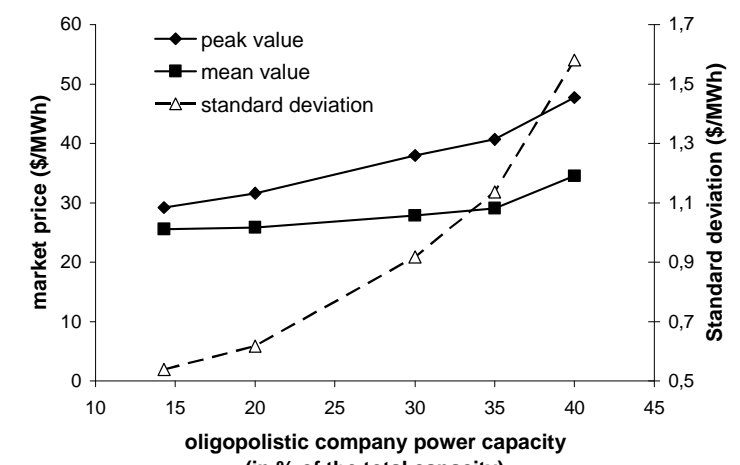

b)

(in \% of the total capacity)

Fig. 5. a) the oligopolistic company profits, in per unit of the profit for the reference case $(865,359 \$)$, the relevant reduction of energy generation, with respect to the cost based UC solution, and b) the average and peak values of the market prices and its standard deviation, for the reference case and for four other cases in which the oligopolistic company has a lower production capacity (namely 35\%, 30\%, 20\% and $14.3 \%$ of the system production capacity).

Fig. 5 shows the oligopolistic company profits, in per unit of the profit for the reference case, the relevant reduction of the energy generation, with respect to the cost based UC solution, and the average and peak values of the market prices and its standard deviation, for the reference case and for other four cases in which the oligopolistic company has a lower production capacity.

\section{CONCLUSIONS}

The most convenient bidding strategies decisions in an electricity market depends on a number of different factors, e.g. production capacity of the company, types of the generating units, number, size and characteristics of the competitors, market rules. Most of these factors are inaccurately known. Therefore, new software tools are, in general, developed in order to build the offers of the generating companies.

This paper has investigated the use of the classical cost-based unit commitment algorithms for the solution of maxim-profit unit commitment problems faced by generating companies in the competitive electricity markets. The paper has considered the cases of competitive generating companies without market power, and the case of an oligopolistic company that has the capability to influence the market price.

By using a unit commitment software, based on the Lagrangian relaxation approach and on a disaggregated
Bundle method for the solution of the dual problem, the paper has shown the minor modifications needed to solve the maximum profit problems and to use the unit commitment software to assist bidding strategy decisions.

\section{ACKNOWLEDGMENT}

The authors gratefully acknowledge the contributions of G. Allegranza and L. Baruzzi for their collaboration in performing the calculations.

\section{REFERENCES}

[1] G.B. Sheblé, Computational auction mechanisms for restructured power industry operation, Kluwer, Norwell, 1999.

[2] C. D. Wolfram, "Strategic bidding in a multiunit auction: an empirical analysis of bids to supply electricity in England and Wales", Rand Journal of Economics, Vol. 29, pp. 703 - 725, 1998.

[3] G. Gross, D. Finlay, and G. Deltas, "Strategic bidding in electricity generation supply markets", in Proc. of IEEE PES Winter Meeting, New York, Vol. 1, pp. $309-315,1999$

[4] J. Garcia, J. Roman, J. Barquin, A. Gonzalez, "Strategic bidding in deregulated power systems", in Proc. of $13^{\text {th }}$ PSCC, Trondheim, June $28^{\text {th }}-$ July $2^{\text {nd }}, 1999$.

[5] J.M. Arroyo and A.J. Conejo, "Optimal response of a thermal unit to an electricity spot market”, IEEE Trans. on Power Systems, Vol. 15, No. 5, pp. 1098-1104, 2000.

[6] F. Wen and A.K. David, "Optimal bidding strategies and modeling of imperfect information among competitive generators", IEEE Trans. on Power Systems, Vol. 16, pp. 15 - 21, 2001.

[7] X. Guan, E. Ni, P.B. Luh, Yu-Chi Ho, "Optimization-based bidding strategies for deregulated electric power markets", in The next generation of electric power unit commitments models, Kluwer, Norwell, 2001.

[8] A.K. David and F. Wen, "Strategic bidding in competitive electricity markets: a literature survey", in Proc. of IEEE PES Summer Meeting, Vol. 4, pp. 2168 - 2173, 2001.

[9] A. Martini, P. Pelacchi, L. Pellegrini, M.V. Cazzol, A. Garzillo, M. Innorta, "A simulation tool for short term electricity markets", in Proc of PICA 2001, May 2001, Sidney, Australia.

[10] M. Benini, M.V. Cazzol, M. Gallanti, A. Gelmini, "Flexible Electricity Market Simulator", in Proc. of World Energy Council, 18th Congress, Buenos Aires, October 2001.

[11] J. Contreras, O. Candiles, J.I. de la Fuente, T. Gomez, "A Cobweb bidding strategy for Competitive Electricity Markets", IEEE Trans. on Power Systems, Vol 17, pp 148-153, February 2002.

[12] J.M. Arroyo and A.J. Conejo, "Optimal response of a power generator to energy, AGC, and reserve pool-based markets", IEEE Trans. on Power Systems, Vol. 17, No. 2, pp. 404-410, 2002.

[13] A. Baillo, M. Ventosa, M. Rivier, A. Ramos, G. Relano, "Bidding in a day-ahead electricity market: a comparison of decomposition techniques", in Proc. of $14^{\text {th }}$ PSCC, Sevilla, 24-28 June 2002.

[14] A.J. Conejo, J. Contreras, J.M. Arroyo and S. de la Torre, "Optimal response of an oligopolistic generating company to a competitive poolbased electric power market", IEEE Trans. on Power Systems, Vol. 17, No. 2, pp. 424-430, 2002.

[15] E. NI and P.B. Luh, "Optimal integrated generation bidding and scheduling with risk management under a deregulated daily power market”, in Proc. of Power Engineering Society Winter Meeting, 2002.

[16] J. W. Bialek, "Gaming the uniform-price spot market: quantitative analysis”, IEEE Trans. on Power Systems, Vol. 17, No. 3, pp. 768-773, 2002.

[17] C.J. Day, B.F. Hobbs, Jong-Shi Pang, "Oligopolistic Competition in power networks: a conjectures supply function approach", IEEE Trans. on Power Systems, Vol. 17, No. 3, pp 597 -607, Aug. 2002.

[18] A.J. Conejo, F.J. Nogales, J.M. Arroyo, "Price-taker bidding strategy under price uncertainty", IEEE Transactions on Power Systems, Vol. 17, No. 4 , pp. $1081-1088,2002$.

[19] A. Borghetti, A. Frangioni, F. Lacalandra, C.A. Nucci, "Lagrangian Heuristics Based on Disaggregated Bundle Methods 
for Hydrothermal Unit Commitment”, IEEE Trans. on Power Systems, Vol. 18, No. 1, pp. 313-323, 2003.

[20] A. Martin., J.I. de la Fuente, "New forecasting method for the residual demand curves using time series (ARIMA) models", in Proc. of PMAPS, Naples, Italy, 2002.

[21] Y. Ermoliev "Stochastic quasi-gradient methods and their applications to systems optimization", Stochastics, 4, pp. 1-37, 1983.

[22] L. Bacaud, C. Lemarechal, A. Renaud, C. Sagastizabal, "Bundle Methods in Stochastic Optimal Power Management: A Disaggregated Approach Using Preconditioners", Computational Optimization and Applications, 20, pp. 227-244, 2001.

[23] V. Petridis, S. Kazarlis and A. Bakirtzis, "Varying Fitness Functions in Genetic Algorithm Constrained Optimization: The Cutting Stock and Unit Commitment Problems", IEEE Trans. on Systems, Man and Cybernetics, part B, vol 28, no 5, October 1998. 